Also available at http://amc.imfm.si

ISSN 1855-3966 (printed edn.), ISSN 1855-3974 (electronic edn.)

ARS MATHEMATICA CONTEMPORANEA 7 (2014) 55-72

\title{
Cayley graphs on nilpotent groups with cyclic commutator subgroup are hamiltonian
}

\author{
Ebrahim Ghaderpour, Dave Witte Morris \\ Department of Mathematics and Computer Science, University of Lethbridge \\ Lethbridge, Alberta, T1K 3M4, Canada
}

Received 27 November 2011, accepted 2 July 2012, published online 22 February 2013

\begin{abstract}
We show that if $G$ is any nilpotent, finite group, and the commutator subgroup of $G$ is cyclic, then every connected Cayley graph on $G$ has a hamiltonian cycle.
\end{abstract}

Keywords: Cayley graph, hamiltonian cycle, nilpotent group, commutator subgroup.

Math. Subj. Class.: 05C25, 05C45

\section{Introduction}

It has been conjectured that every connected Cayley graph has a hamiltonian cycle. See $[4,10,11,14,15,17]$ for references to some of the numerous results on this problem that have been proved in the past forty years, including the following theorem that is the culmination of papers by Marušič [12], Durnberger [5, 6], and Keating-Witte [9]:

Theorem 1.1 (D. Marušič, E. Durnberger, K. Keating, and D. Witte, 1985). Let $G$ be a nontrivial, finite group. If the commutator subgroup $[G, G]$ of $G$ is cyclic of prime-power order, then every connected Cayley graph on $G$ has a hamiltonian cycle.

It is natural to try to prove a generalization that only assumes the commutator subgroup is cyclic, without making any restriction on its order, but that seems to be an extremely difficult problem: at present, it is not even known whether all connected Cayley graphs on dihedral groups have hamiltonian cycles. (See [1,2] and [15, Cor. 5.2] for the main results that have been proved for dihedral groups.) In this paper, we replace the assumption on the order of $[G, G]$ with the rather strong assumption that $G$ is nilpotent:

Theorem 1.2. Let $G$ be a nontrivial, finite group. If $G$ is nilpotent, and the commutator subgroup of $G$ is cyclic, then every connected Cayley graph on $G$ has a hamiltonian cycle.

E-mail addresses: Ebrahim.Ghaderpoor@uleth.ca (Ebrahim Ghaderpour), Dave.Morris@uleth.ca, http://people.uleth.ca/ dave.morris/ (Dave Witte Morris) 
The proof of this theorem is based on a variant of the method of D. Marušič [12] that established theorem 1.1 (cf. [9, Lem. 3.1]).

Remark 1.3. Here are some previous results on the hamiltonicity of the Cayley graph $\operatorname{Cay}(G ; S)$ when $G$ is nilpotent:

1. Assume $G$ is nilpotent, the commutator subgroup of $G$ is cyclic, and $S$ has only two elements. Then a hamiltonian cycle in $\operatorname{Cay}(G ; S)$ was found in $[9, \S 6]$ (see proposition 3.16). The present paper generalizes this by eliminating the restriction on the cardinality of the generating set $S$.

2. For Cayley graphs on nilpotent groups (without any assumption on the commutator subgroup) it was recently shown that if the valence is at most 4 , then there is a hamiltonian path (see [13]).

3. Every nilpotent group is a direct product of $p$-groups. For $p$-groups, it is known that every Cayley graph has a hamiltonian cycle ([16], see theorem 3.13). Unfortunately, we do not know how to extend this to direct products.

4. Every abelian group is nilpotent. It is well known (and easy to prove) that Cayley graphs on abelian groups always have hamiltonian cycles. In fact, there is usually a hamiltonian path from any vertex to any other vertex (see [3]).

Acknowledgments. We thank Dragan Marušič and Mohammad Reza Salarian for their comments that encouraged this line of research. We also thank an anonymous referee for reading an earlier version of the paper very carefully, and providing many corrections and helpful comments.

\section{Assumptions and notation}

We begin with some standard notation:

Notation 2.1. Let $G$ be a group, and let $S$ be a subset of $G$.

- $\operatorname{Cay}(G ; S)$ denotes the Cayley graph of $G$ with respect to $S$. Its vertices are the elements of $G$, and there is an edge joining $g$ to $g s$ for every $g \in G$ and $s \in S$.

- $G^{\prime}=[G, G]$ denotes the commutator subgroup of $G$.

- $S^{r}=\left\{s^{r} \mid s \in S\right\}$ for any $r \in \mathbb{Z}$. (Similarly, $G^{r}=\left\{g^{r} \mid g \in G\right\}$.)

- $S^{ \pm 1}=S \cup S^{-1}$.

- \#S is the cardinality of $S$.

Note that if $S$ happens to be a cyclic subgroup of $G$, then $S^{r}$ is a subgroup of $S$.

We now fix notation designed specifically for our proof of theorem 1.2:

\section{Notation 2.2.}

- $G$ is a nilpotent, finite group,

- $N$ is a cyclic, normal subgroup of $G$ that contains $G^{\prime}$,

- $g \mapsto \bar{g}$ is the natural homomorphism from $G$ to $G / N=\bar{G}$,

- $S=\left\{\sigma_{1}, \sigma_{2}, \ldots, \sigma_{\ell}\right\}$ is a subset of $G$, such that 
$\circ \bar{S}$ is a minimal generating set for $\bar{G}$, and

$\circ \ell=\# S=\# \bar{S} \geq 2$,

The minimality of $\bar{S}$ implies that $e \notin S$, and that if $s \in S$ and $|s| \geq 3$, then $s^{-1} \notin S$.

- $S_{k}=\left\{\sigma_{i} \mid i \leq k\right\}$ for $1 \leq k \leq \ell$,

- $G_{k}=\left\langle S_{k}\right\rangle N$, and

- $m_{k}=\left|\overline{G_{k}}: \overline{G_{k-1}}\right|$.

\section{Definition 2.3.}

- If $\left(s_{i}\right)_{i=1}^{n}$ is a sequence of elements of $S^{ \pm 1}$ and $\bar{g} \in \bar{G}$, we use $\bar{g}\left(s_{i}\right)_{i=1}^{n}$ to denote the walk in $\operatorname{Cay}(\bar{G} ; \bar{S})$ that visits (in order) the vertices

$$
\bar{g}, \overline{g s_{1}}, \overline{g s_{1} s_{2}}, \ldots, \overline{g s_{1} s_{2} \cdots s_{n}} .
$$

- If $C=\bar{g}\left(s_{i}\right)_{i=1}^{n}$ is any oriented cycle in $\operatorname{Cay}(\bar{G} ; \bar{S})$, its voltage is $\prod_{i=1}^{n} s_{i}$. This is an element of $N$, and it may be denoted $\Pi C$.

- For $S_{0} \subset S$, we say the walk $\bar{g}\left(s_{i}\right)_{i=1}^{n}$ covers $S_{0}^{ \pm 1}$ if it contains an oriented edge labeled $s$ and a (different) oriented edge labeled $s^{-1}$, for every $s \in S_{0}$. (That is, there exist $i, j$ with $i \neq j$, such that $s_{i}=s$ and $s_{j}=s^{-1}$. When $|s|=2$, this means $s_{i}=s_{j}=s$.)

- $\mathcal{V}_{k}$ is the set of voltages of oriented hamiltonian cycles in the graph $\operatorname{Cay}\left(\overline{G_{k}} ; \overline{S_{k}}\right)$ that cover $S_{k}^{ \pm 1}$.

Notation 2.4. For $k \in \mathbb{Z}^{+}$, we use $\left(s_{1}, \ldots, s_{n}\right)^{k}$ to denote the concatenation of $k$ copies of the sequence $\left(s_{1}, \ldots, s_{n}\right)$. Abusing notation, we often write $s^{k}$ and $s^{-k}$ for

$$
(s)^{k}=(s, s, \ldots, s) \text { and }\left(s^{-1}\right)^{k}=\left(s^{-1}, s^{-1}, \ldots, s^{-1}\right),
$$

respectively. Furthermore, we often write $\left(\left(s_{1}, \ldots, s_{m}\right),\left(t_{1}, \ldots, t_{n}\right)\right)$ to denote the concatenation $\left(s_{1}, \ldots, s_{m}, t_{1}, \ldots, t_{n}\right)$. For example, we have

$$
\left(\left(a^{3}, b\right)^{2}, c^{-2}\right)^{2}=\left(a, a, a, b, a, a, a, b, c^{-1}, c^{-1}, a, a, a, b, a, a, a, b, c^{-1}, c^{-1}\right)
$$

The following well-known, elementary observation is the foundation of our proof:

\section{Lemma 2.5 ("Factor Group Lemma” [17, §2.2]). Suppose}

- $N$ is a cyclic, normal subgroup of $G$,

- $C=\bar{g}\left(s_{i}\right)_{i=1}^{n}$ is a hamiltonian cycle in $\operatorname{Cay}(\bar{G} ; \bar{S})$, and

- the voltage $\Pi C$ generates $N$.

Then $\left(s_{1}, \ldots, s_{n}\right)^{|N|}$ is a hamiltonian cycle in $\operatorname{Cay}(G ; S)$.

With this in mind, we let $N=G^{\prime}$, and we would like to find a hamiltonian cycle in Cay $(\bar{G} ; \bar{S})$ whose voltage generates $N$. In almost all cases, we will do this by induction on 
$\ell=\# S$, after substantially strengthening the induction hypothesis. Namely, we consider the following assertion $\left(\alpha_{k}^{\epsilon}\right)$ for $2 \leq k \leq \ell$ and $\epsilon \in\{1,2\}$ :

there exists $h_{k} \in N$, such that, for every $x \in N$,

$\left(\mathcal{V}_{k} \cap h_{k}\left(G_{k}^{\prime}\right)^{\epsilon}\right) x$ contains a generator of

a subgroup of $N$ that contains $\left(G_{k}^{\prime}\right)^{\epsilon}$.

For $\epsilon=2$, we also consider the following slightly stronger condition, which we call $\alpha_{k}^{2+}$ :

$$
\alpha_{k}^{2} \text { holds, and }\left\langle h_{k},\left(G_{k}^{\prime}\right)^{2}\right\rangle \text { contains } G_{k}^{\prime} \text {. }
$$

Lemma 2.6. Let $N=G^{\prime}$. If either $\alpha_{\ell}^{1}$ or $\alpha_{\ell}^{2+}$ holds, then there is a hamiltonian cycle in $\operatorname{Cay}(\bar{G} ; \bar{S})$ whose voltage generates $N$.

Proof. Note that $G_{\ell}^{\prime}=G^{\prime}=N$. Since $\mathcal{V}_{\ell}$ consists of voltages of hamiltonian cycles in $\operatorname{Cay}(\bar{G} ; \bar{S})$, it suffices to find an element of $\mathcal{V}_{\ell}$ that generates $G_{\ell}^{\prime}$.

If we assume $\alpha_{\ell}^{1}$, then the desired conclusion is immediate, by taking $x=e$ in that assertion.

Similarly, if we assume $\alpha_{\ell}^{2+}$, then taking $x=e$ in $\alpha_{\ell}^{2}$ tells us that some element $\gamma$ of $\mathcal{V}_{\ell} \cap h_{\ell}\left(G_{\ell}^{\prime}\right)^{2}$ generates a subgroup of $N$ that contains $\left(G_{\ell}^{\prime}\right)^{2}$. Then, since $\gamma \in\left(G_{\ell}^{\prime}\right)^{2} h_{\ell}$, and $\left\langle h_{\ell},\left(G_{\ell}^{\prime}\right)^{2}\right\rangle$ contains $G_{\ell}^{\prime}$, we have

$$
N \supset\langle\gamma\rangle=\left\langle\gamma,\left(G_{\ell}^{\prime}\right)^{2}\right\rangle=\left\langle h_{\ell},\left(G_{\ell}^{\prime}\right)^{2}\right\rangle \supset G_{\ell}^{\prime}=N .
$$

\section{Remark 2.7.}

1. If $\left|G_{k}^{\prime}\right|$ is odd, then $\left(G_{k}^{\prime}\right)^{2}=G_{k}^{\prime}$, so we have $\alpha_{k}^{1} \Leftrightarrow \alpha_{k}^{2} \Leftrightarrow \alpha_{k}^{2+}$ in this case. Thus, the parameter $\epsilon$ is only of interest when $\left|G^{\prime}\right|$ is even.

2. It is not difficult to see that $\alpha_{k}^{1} \Rightarrow \alpha_{k}^{2+}$, but we do not need this fact.

Our proof of $\alpha_{\ell}^{1}$ or $\alpha_{\ell}^{2+}$ is by induction on $k$. Here is the outline:

I. We prove a base case of the induction: $\alpha_{2}^{2}$ is usually true (see proposition 4.1).

II. We prove an induction step: under certain conditions, $\alpha_{k}^{1} \Rightarrow \alpha_{k+1}^{1}$ and $\alpha_{k}^{2+} \Rightarrow \alpha_{k+1}^{2+}$ (see proposition 5.4).

III. We prove $\alpha_{\ell}^{1}$ or $\alpha_{\ell}^{2+}$ is usually true, by bridging the gap between $\alpha_{2}^{2}$ and either $\alpha_{3}^{1}$ or $\alpha_{3}^{2+}$, and then applying the induction step (see corollary 6.1 and proposition 6.2).

\section{Preliminaries}

\section{A Remarks on voltage}

Remark 3.1. By definition, it is clear that all translates of an oriented cycle $C$ in Cay $(\bar{G} ; \bar{S})$ have the same voltage. That is,

$$
\Pi\left(\bar{g}\left(s_{i}\right)_{i=1}^{n}\right)=\Pi\left(\left(s_{i}\right)_{i=1}^{n}\right) .
$$

Remark 3.2. If $|N|$ is square-free (which is usually the case in this paper), then $N$ is contained in the center of $G$ (because $N$ is the direct product of normal subgroups of prime order, and it is well known that those are all in the center [8, Thm. 4.3.4]). In this situation, 
the voltage of a cycle in $\operatorname{Cay}(\bar{G} ; \bar{S})$ is independent of the starting point that is chosen for its representation. That is, if $\left(t_{i}\right)_{i=1}^{n}$ is a cyclic rotation of $\left(s_{i}\right)_{i=1}^{n}$, so there is some $r \in\{0,1,2, \ldots, n\}$ with $t_{i}=s_{i+r}$ for all $i$ (where subscripts are read modulo $n$ ), then

$$
\Pi\left(t_{i}\right)_{i=1}^{n}=s_{r+1} s_{r+2} \cdots s_{n} s_{1} s_{2} \cdots s_{r}=\left(s_{1} \cdots s_{r}\right)^{-1}\left(\Pi\left(s_{i}\right)_{i=1}^{n}\right) s_{1} \cdots s_{r}=\Pi\left(s_{i}\right)_{i=1}^{n},
$$

because $\Pi\left(s_{i}\right)_{i=1}^{n} \in N \subset Z(G)$.

The following observation is useful for calculating voltages:

Lemma 3.3. If $a, b \in G, G^{\prime} \subset Z(G)$, and $p_{1}, q_{1}, \ldots, p_{r}, q_{r} \in \mathbb{Z}$, then

$$
a^{p_{1}} b^{q_{1}} a^{p_{2}} b^{q_{2}} \cdots a^{p_{r}} b^{q_{r}}=a^{p_{1}+\cdots+p_{r}} b^{q_{1}+\cdots+q_{r}}[a, b]^{-\Sigma},
$$

where $\Sigma=\sum_{i>j} p_{i} q_{j}$.

Proof. The desired conclusion is easily proved by induction on $r$, using the fact that, since $G^{\prime} \subset Z(G)$, we have $\left[a^{p}, b^{q}\right]=[a, b]^{p q}$ for $p, q \in \mathbb{Z}$ [7, Lem. 2.2(i)].

\section{B Facts from group theory}

Lemma 3.4. If $\left|G_{k}^{\prime}\right|$ is square-free, then $\left|G_{k}^{\prime} / G_{k-1}^{\prime}\right|$ is a divisor of both $\left|\overline{G_{k-1}}\right|$ and $\left|\overline{G_{k}} / \overline{G_{k-1}}\right|$.

Proof. We may assume $k=\ell$, so $G=G_{k}$. Let $p$ be a prime factor of $\left|G^{\prime} / G_{k-1}^{\prime}\right|$, let $P$ be the Sylow $p$-subgroup of $G$, and let $\varphi: G \rightarrow P$ be the natural projection. Since $\left|G^{\prime}\right|$ is square-free, it suffices to show that $\left|\overline{G_{k-1}}\right|$ and $\left|\overline{G_{k}} / \overline{G_{k-1}}\right|$ are divisible by $p$.

We may assume $\left|G^{\prime}\right|=p$ and $G_{k-1}^{\prime}=\{e\}$, by modding out the unique subgroup of index $p$ in $G^{\prime}$. Therefore $\varphi\left(G_{k-1}\right)$ is abelian, so it is a proper subgroup of $P$. Since $G^{\prime}=P^{\prime} \subset \Phi(P)$, this implies $\varphi\left(G_{k-1}\right) G^{\prime}$ is a proper subgroup of $P$, so its index is divisible by $p$. Hence $\left|\bar{G} / \overline{G_{k-1}}\right|$ is divisible by $p$.

There must be some $t \in S_{k-1}$, such that $\left[\sigma_{k}, t\right]$ is nontrivial. Hence $\varphi(t) \notin Z(G) \supset G^{\prime}$, so $p$ is a divisor of $|\overline{\varphi(t)}|$, which is a divisor of $\left|\overline{G_{k-1}}\right|$.

The following fact is well known and elementary, but we do not know of a reference in the literature. It relies on our assumption that $G^{\prime}$ is cyclic.

Lemma 3.5. We have $\langle[s, t] \mid s, t \in S\rangle=G^{\prime}$ if $N \subset Z(G)$.

Proof. Let $H=\langle[s, t] \mid s, t \in S\rangle$. Then $H$ is a normal subgroup of $G$, because every subgroup of a cyclic, normal subgroup is normal. In $G / H$, every element of $S$ commutes with all of the other elements of $S$ (and with all of $N$ ), so $G / H$ is abelian. Hence $G^{\prime} \subset$ $H$.

\section{C Elementary facts about cyclic groups of square-free order}

Lemma 3.6. Assume $|N|$ is square-free, and $H$ and $K$ are two subgroups of $N$. Then:

1. There is a unique subgroup $K^{\perp}$ of $N$, such that $N=K \times K^{\perp}$.

2. $K^{\perp}$ is a normal subgroup of $G$.

3. $K \subseteq H$ iff $\underline{H}=\underline{N}$ in $\underline{G}=G / K^{\perp}$. 
Proof. (1) Since $N$ is cyclic, it has a unique subgroup of any order dividing $|N|$; let $K^{\perp}$ be the subgroup of order $|N / K|$. Since $|N|$ is square-free, we have $\operatorname{gcd}\left(|K|,\left|K^{\perp}\right|\right)=1$, so $N=K \times K^{\perp}$.

(2) It is well known that every subgroup of a cyclic, normal subgroup is normal (because no other subgroup of $N$ has the same order).

(3) We prove only the nontrivial direction. Since $\underline{H}=\underline{N}$, we know that $|K|=|\underline{N}|$ is a divisor of $|H|$. So $|H|$ has a subgroup whose order is $|K|$. Since $K$ is the only subgroup of $N$ with this order, we must have $K \subseteq H$.

Remark 3.7. When we want to show that some subgroup $H$ of $N$ contains some other subgroup $K$, Lemma 3.6 often allows us to assume $K=N$ (by modding out $K^{\perp}$ ), which means we wish to prove $H=N$.

Lemma 3.8. Suppose

- $\gamma$ is a generator of $N$,

- $x \in N$, and

- $a \geq \max (|N|, 5)$.

Then, for some $i$ with $1 \leq i \leq\lfloor(a-1) / 2\rfloor$, we have $N^{2} \subseteq\left\langle\gamma^{-2 i} x\right\rangle$.

Proof. Write $x=\gamma^{h}$, where $1 \leq h \leq|N|$, choose $r \in\{1,2\}$ such that $h-r$ is even, and let

$$
i=\left\{\begin{array}{cl}
r & \text { if } h \in\{1,2\}, \\
(h-r) / 2 & \text { if } h>2 .
\end{array}\right.
$$

Then $h-2 i \in\{ \pm r\} \subset\{ \pm 1, \pm 2\}$, so $N^{2} \subseteq\left\langle\gamma^{h-2 i}\right\rangle=\left\langle\gamma^{-2 i} x\right\rangle$.

\section{Lemma 3.9. If}

- $N$ is a cyclic group of square-free order,

- $m \geq|N|$,

- $k \geq 2$,

- $T=\left\{\gamma_{1}, \ldots, \gamma_{k}\right\}$ generates $N$,

- $h \in N$, and

- $\operatorname{Cay}(N ; T)$ is not bipartite,

then we may choose a sequence $\left(j_{i}\right)_{i=1}^{m-1}$ of elements of $\{1,2, \ldots, k\}$, and $\gamma_{i}^{*} \in\left\{\gamma_{j_{i}}^{ \pm 1}\right\}$ for each $i$, such that $\gamma_{i+1}^{*}=\gamma_{i}^{*}$ whenever $j_{i+1}=j_{i}$, and

$$
\left\langle h \gamma_{1}^{*} \gamma_{2}^{*} \cdots \gamma_{m-1}^{*}\right\rangle=N
$$

Proof. Let us assume $|N|>3$. (The smaller cases are very easy to address individually.)

We begin by finding $\gamma_{1}^{*}, \gamma_{2}^{*}, \ldots, \gamma_{m-1}^{*} \in T^{ \pm 1}$, such that $\left\langle h \gamma_{1}^{*} \gamma_{2}^{*} \cdots \gamma_{m-1}^{*}\right\rangle$ contains $N^{2}$ (or $N$, if appropriate), but without worrying about the requirement that $\gamma_{i+1}^{*}=\gamma_{i}^{*}$ whenever $j_{i+1}=j_{i}$.

Let $\gamma$ be a generator of $N$, and assume $h^{-1} \gamma \neq e$ (by replacing $\gamma$ with its inverse, if necessary). Since $\operatorname{Cay}(N ; T)$ is not bipartite, there is a walk $\left(\gamma_{i}^{*}\right)_{i=1}^{r}$ from $e$ to $h^{-1} \gamma$, such that $r \equiv m-1(\bmod 2)$. 
We now show the walk can be chosen to satisfy the additional constraint that $r<|N|$ (so $r \leq m-1)$. We know that $\operatorname{Cay}(N ; T)$ has a hamiltonian cycle $C$ (since $N$ is abelian). Since $\operatorname{Cay}(N ; T)$ is not bipartite, $C$ must have a chord $L$ of even length. We may assume one endpoint of $L$ is $e$, since $\operatorname{Cay}(N ; T)$ is vertex transitive. Let $z$ be the other endpoint of $L$. Being a hamiltonian cycle, $C$ can be written as the union of two edge-disjoint paths from $e$ to $h^{-1} \gamma$. Let $P$ be the one of these paths that contains a subpath of even length from $e$ to $z$, and let $\widehat{P}$ be obtained from $P$ by replacing this subpath with the edge $L$. Then $P$ and $\widehat{P}$ are two paths from $e$ to $h^{-1} \gamma$. Both have length less than $|N|$, and their lengths are of opposite parity.

Now

$$
h \gamma_{1}^{*} \gamma_{2}^{*} \cdots \gamma_{r}^{*}\left(\gamma_{1} \gamma_{1}^{-1}\right)^{(m-1-r) / 2}=\gamma \text { generates } N,
$$

as desired.

To complete the proof of the lemma, we modify the above sequence $\gamma_{1}^{*}, \gamma_{2}^{*}, \ldots, \gamma_{m-1}^{*}$ to satisfy the condition that $\gamma_{i+1}^{*}=\gamma_{i}^{*}$ whenever $j_{i+1}=j_{i}$. First of all, since $N$ is commutative, we may collect like terms, and thereby write

$$
\gamma_{1}^{*} \gamma_{2}^{*} \cdots \gamma_{m-1}^{*}=\gamma_{1}^{m_{1}} \gamma_{2}^{m_{2}} \cdots \gamma_{k}^{m_{k}} \gamma_{1}^{-n_{1}} \gamma_{2}^{-n_{2}} \cdots \gamma_{k}^{-n_{k}}
$$

where $m_{1}+\cdots+m_{k}+n_{1}+\cdots+n_{k}=m-1$. Notice that if $m_{k}$ and $n_{1}$ are both nonzero, then no occurrence of $\gamma_{i}$ is immediately followed by $\gamma_{i}^{-1}$; so we have $\gamma_{i+1}^{*}=\gamma_{i}^{*}$ whenever $j_{i+1}=j_{i}$, as desired. Therefore, by permuting $\gamma_{1}, \ldots, \gamma_{k}$, we may assume $m_{i}=n_{i}=0$ for all $i>1$. Also, we may assume $m_{1}$ and $n_{1}$ are both nonzero, for otherwise we have $\gamma_{i}^{*}=\gamma_{j}^{*}$ for all $i$ and $j$. Then, since $\gamma_{1} \gamma_{1}^{-1}=\gamma_{2} \gamma_{2}^{-1}$, we have

$$
\gamma_{1}^{*} \gamma_{2}^{*} \cdots \gamma_{m-1}^{*}=\gamma_{1}^{m_{1}} \gamma_{1}^{-n_{1}}=\gamma_{1}^{m_{1}-1} \gamma_{2} \gamma_{1}^{-\left(n_{1}-1\right)} \gamma_{2}^{-1}
$$

We can assume $n_{1} \geq m_{1}$ (by replacing $\gamma_{1}$ with its inverse, if necessary). Then

$$
n_{1} \geq\left\lceil\frac{m-1}{2}\right\rceil \geq\left\lceil\frac{|N|-1}{2}\right\rceil \geq 2,
$$

so this new representation of the same product satisfies the condition that $\gamma_{i}$ is never immediately followed by $\gamma_{i}^{-1}$. This completes the proof.

Corollary 3.11. Assume $|N|$ is square-free and $k \geq 2$ (and $\epsilon \in\{1,2\}$, as usual). For convenience, let $m=m_{k+1}$ and $a=\sigma_{k+1}$. If $h \in N$, then there exists a sequence $\left(s_{i}\right)_{i=1}^{m-1}$ of elements of $S_{k}$, and $s_{i}^{*} \in\left\{s_{i}^{ \pm 1}\right\}$ for each $i$, such that $s_{i+1}^{*}=s_{i}^{*}$ whenever $s_{i+1}=s_{i}$, and

$$
\left\langle h \prod_{i=1}^{m-1}\left[a, s_{i}^{*}\right],\left(G_{k}^{\prime}\right)^{\epsilon}\right\rangle \text { contains } \begin{cases}G_{k+1}^{\prime} & \text { if there exist } s, s^{\prime} \in S_{k}, \text { such that } \\ & |[a, s]| \text { is even and }\left|\left[a, s^{\prime}\right]\right| \text { is odd, } \\ \left(G_{k+1}^{\prime}\right)^{\epsilon} & \text { if }\left|G_{k+1}^{\prime} / G_{k}^{\prime}\right| \text { is odd } \\ \left(G_{k+1}^{\prime}\right)^{2} & \text { otherwise. }\end{cases}
$$

Proof. For each $s \in S_{k}$, let $\gamma_{s}=[a, s]$. Also, let

$$
T=\left\{\gamma_{s} \mid s \in S_{k}\right\} \subseteq G_{k+1}^{\prime} \subseteq N .
$$


Let

$$
\widehat{\epsilon}= \begin{cases}1 & \text { if there exist } t, t^{\prime} \in T, \text { such that }|t| \text { is even and }\left|t^{\prime}\right| \text { is odd } \\ \epsilon & \text { if }\left|G_{k+1}^{\prime} / G_{k}^{\prime}\right| \text { is odd } \\ 2 & \text { otherwise. }\end{cases}
$$

Lemma 3.6 allows us to assume $\left(G_{k+1}^{\prime}\right)^{\widehat{\epsilon}}=N$, by modding out $\left(\left(G_{k+1}^{\prime}\right)^{\widehat{\epsilon}}\right)^{\perp}$. We can also assume $\left(G_{k}^{\prime}\right)^{\epsilon}$ is trivial, by modding it out.

We claim that $\langle T\rangle=N$. We have

$$
\begin{aligned}
\left\langle T, G_{k}^{\prime}\right\rangle & \supseteq\left\langle\left\{\left[\sigma_{k+1}, s\right] \mid s \in S_{k}\right\},\left\{[s, t] \mid s, t \in S_{k}\right\}\right\rangle \\
& =\left\langle\left\{[s, t] \mid s, t \in S_{k+1}\right\}\right\rangle \\
& =G_{k+1}^{\prime} \\
& =N .
\end{aligned}
$$

(see Lemma 3.5)

Since $\left(G_{k}^{\prime}\right)^{\epsilon}$ is trivial, this implies $|N:\langle T\rangle| \leq \epsilon$. Thus, if the claim is not true, must have $|N:\langle T\rangle|=\epsilon=2$. In particular, $|N|$ is even. Since $\left(G_{k+1}^{\prime}\right)^{\widehat{\epsilon}}=N$, we conclude that $\widehat{\epsilon}=1$, so the definition of $\widehat{\epsilon}$ (together with the fact that $\epsilon=2$ ) implies that $T$ contains an element of even order. So $|\langle T\rangle|$ is even, which contradicts the fact that $|N:\langle T\rangle|=2$ is even (and $|N|$ is square-free). This completes the proof of the claim.

We also claim that $\operatorname{Cay}(N ; T)$ is not bipartite. We may assume $|N|$ is even (for otherwise the claim is obvious). Since $\left(G_{k+1}^{\prime}\right)^{\widehat{\epsilon}}=N$, this implies $\widehat{\epsilon}=1$. However, the claim is also obviously true if there exist $t, t^{\prime} \in T$, such that $|t|$ is even and $\left|t^{\prime}\right|$ is odd. Hence, we may assume $\left|G_{k+1}^{\prime} / G_{k}^{\prime}\right|$ is odd, and $\epsilon=\widehat{\epsilon}=1$. Since $\left(G_{k}^{\prime}\right)^{\epsilon}$ is trivial, this implies $\left|G_{k}^{\prime}\right|$ is odd, which contradicts the fact that $|N|$ is even. This completes the proof of the claim.

The desired conclusion is now immediate from Lemma 3.9 (since $\gamma_{s}=[a, s]$ and $\left.\left(\gamma_{s}\right)^{-1}=\left[a, s^{-1}\right]\right)$

\section{D Results from [9] and [16]}

The following result from [16] allows us to assume $G$ is not a 3-group. (Since we always assume that $G^{\prime}$ is cyclic, a short proof of the special case we need can be found in [15, Thm. 6.1].)

Theorem 3.13 (Witte [16]). If $|G|$ is a power of some prime $p$, then every connected Cayley graph on $G$ has a hamiltonian cycle.

The following simple observation usually allows us to assume $|N|$ is square-free.

Lemma 3.14 ([9, Lem. 3.2]). Let $\underline{G}=G / \Phi(N)$, where $\Phi(N)$ is the Frattini subgroup of $N[8, \S 10.4]$. Then:

1. $|\underline{N}|$ is square-free, and

2. if there is a hamiltonian cycle in $\operatorname{Cay}(\underline{G} / \underline{N} ; S)$ whose voltage generates $\underline{N}$, then there is a hamiltonian cycle in $\operatorname{Cay}(G / N ; S)$ whose voltage generates $N$.

Lemma 3.15 (Keating-Witte [9, Case 6.1]). If $\left|\overline{G_{2}}\right|$ is even, then Cay $\left(\overline{G_{2}} ; \overline{S_{2}}\right)$ has a hamiltonian cycle whose voltage is a generator of $G_{2}^{\prime}$. 
Proof. For the reader's convenience, we provide a proof. We may assume $\left|\overline{\sigma_{1}}\right|$ is even (by interchanging $\sigma_{1}$ and $\sigma_{2}$ if necessary). For convenience, let $n=\left|\overline{\sigma_{1}}\right|$ and $m=m_{2}$. Then

$$
\left(\sigma_{2}^{m-1},\left(\sigma_{1}, \sigma_{2}^{-(m-2)}, \sigma_{1}, \sigma_{2}^{m-2}\right)^{(n-2) / 2}, \sigma_{1}, \sigma_{2}^{-(m-1)}, \sigma_{1}^{-(n-1)}\right)
$$

is a hamiltonian cycle in $\mathrm{Cay}\left(\overline{G_{2}} ; \overline{S_{2}}\right)$.

Lemma 3.14 allows us to assume $\left|G_{2}^{\prime}\right|$ is square-free, which implies $G_{2}^{\prime}$ is in the center of $G_{2}$ (see remark 3.2). Also, from Lemma 3.4, we know that $\left|\left[\sigma_{1}, \sigma_{2}\right]\right|$ is a divisor of both $m$ and $n$. Therefore

$$
\begin{aligned}
& \left(\sigma_{1} \sigma_{2}^{-(m-2)} \sigma_{1} \sigma_{2}^{m-2}\right)^{(n-2) / 2}=\left(\sigma_{1}^{2}\left[\sigma_{1}, \sigma_{2}^{m-2}\right]\right)^{(n-2) / 2} \\
& =\sigma_{1}^{n-2}\left[\sigma_{1}, \sigma_{2}\right]^{(m-2)(n-2) / 2}=\sigma_{1}^{n-2}\left[\sigma_{1}, \sigma_{2}\right]^{2}
\end{aligned}
$$

and

$$
\left[\sigma_{2}^{-(m-1)}, \sigma_{1}^{-(n-1)}\right]=\left[\sigma_{2}, \sigma_{1}\right]^{(m-1)(n-1)}=\left[\sigma_{2}, \sigma_{1}\right]=\left[\sigma_{1}, \sigma_{2}\right]^{-1},
$$

so the voltage of this cycle is

$$
\begin{aligned}
\sigma_{2}^{m-1} & \left(\sigma_{1} \sigma_{2}^{-(m-2)} \sigma_{1} \sigma_{2}^{m-2}\right)^{(n-2) / 2} \sigma_{1} \sigma_{2}^{-(m-1)} \sigma_{1}^{-(n-1)} \\
& =\sigma_{2}^{m-1}\left(\sigma_{1}^{n-2}\left[\sigma_{1}, \sigma_{2}\right]^{2}\right) \sigma_{1} \sigma_{2}^{-(m-1)} \sigma_{1}^{-(n-1)} \\
& =\sigma_{2}^{m-1} \sigma_{1}^{n-1} \sigma_{2}^{-(m-1)} \sigma_{1}^{-(n-1)}\left[\sigma_{1}, \sigma_{2}\right]^{2} \\
& =\left[\sigma_{2}^{-(m-1)}, \sigma_{1}^{-(n-1)}\right]\left[\sigma_{1}, \sigma_{2}\right]^{2} \\
& =\left[\sigma_{1}, \sigma_{2}\right],
\end{aligned}
$$

which generates $G_{2}^{\prime}$ (see Lemma 3.5).

The following result allows us to assume $\ell \geq 3$.

Proposition 3.16 (Keating-Witte $[9, \S 6])$. If $\ell=2$ and $N=G^{\prime}$, then $\operatorname{Cay}(G ; S)$ has a hamiltonian cycle.

Proof. For the reader's convenience, we provide a proof (using the main result of Section 4 below). We may assume $\left|G / G^{\prime}\right|$ is odd, for otherwise a hamiltonian cycle is obtained by combining Lemma 3.15 with the Factor Group Lemma (2.5). We may also assume that $|G|$ is not a power of 3 , for otherwise theorem 3.13 applies. This implies it is not the case that $|\bar{s}|=3$ for every $s \in S$.

If $\left|G^{\prime}\right|$ is square-free, then proposition 4.1 tells us that $\alpha_{2}^{2}$ is true. Also, since $\left|G / G^{\prime}\right|$ is odd, we know $\left|G^{\prime}\right|$ is odd (cf. Lemma 3.4), so $\alpha_{2}^{2}$ implies that $\alpha_{2}^{1}$ is true (see remark 2.7(1)). Therefore, the Factor Group Lemma (2.5) provides a hamiltonian cycle in $\operatorname{Cay}(G ; S)$ (see Lemma 2.6). Then Lemma 3.14 tells us there is a hamiltonian cycle even without the assumption that $\left|G^{\prime}\right|$ is square-free.

\section{Base case of the inductive construction}

Recall that the condition $\alpha_{k}^{\epsilon}$ is defined in Section 2.

Proposition 4.1 (cf. [9, Case 6.2]). Assume $|N|$ is square-free (and $\ell \geq 2$ ). Then $\alpha_{2}^{2}$ is true unless $\left|G_{2}^{\prime}\right|=m_{2}=\left|\overline{\sigma_{1}}\right|=\left|\overline{\sigma_{2}}\right|=3$. 
Proof. For convenience, let

$$
a=\sigma_{1}, b=\sigma_{2}, \text { and } m=m_{2},
$$

and define $r$ by

$$
\bar{b}^{m}=\bar{a}^{r} \text { and } 0<r \leq|\bar{a}| .
$$

We may assume:

- $\ell=2$, so $S=S_{2}=\{a, b\}$ and $G=G_{2}$.

- $\left(G^{\prime}\right)^{2}$ is nontrivial. (Otherwise, the condition about generating $\left(G^{\prime}\right)^{2}$ is automatically true, so it suffices to show $\mathcal{V}_{2} \neq \emptyset$, which is easy.)

- Either $|\bar{a}|$ is even, or $m$ is odd (by interchanging $\sigma_{1}$ and $\sigma_{2}$ if necessary).

- $|\bar{a}| \neq 3$ (by interchanging $\sigma_{1}$ and $\sigma_{2}$ if necessary: if $\left|\overline{\sigma_{1}}\right|=\left|\overline{\sigma_{2}}\right|=3$, then $m=3$ and, from Lemma 3.4, we also have $\left|G^{\prime}\right|=3$, which means we are in a case in which the statement of the proposition does not make any claim).

- $r \geq|\bar{a}| / 2$ (by replacing $a$ with its inverse if necessary).

Note that $\left|G^{\prime}\right|$ is a divisor of both $|\bar{a}|$ and $m$ (see Lemma 3.4). Since $\left(G^{\prime}\right)^{2}$ is nontrivial, this implies that $|\bar{a}|$ and $m$ both have at least one odd prime divisor.

Case 1. Assume $m=3$. Since $\left|G^{\prime}\right|$ is a divisor of $m$, we must have $\left|G^{\prime}\right|=3$, so $|\bar{a}|$ must be divisible by 3 . Then, since $|\bar{a}| \neq 3$, we must have $|\bar{a}| \geq 6$. Furthermore, by applying Lemma 3.4 with $a$ and $b$ interchanged, we see that $|\bar{G} /\langle\bar{b}\rangle|$ is also divisible by $\left|G^{\prime}\right|=3$, which means that $r$ is divisible by 3 .

We claim that it suffices to find two elements $\gamma_{1}, \gamma_{2} \in \mathcal{V}_{2}$, such that $\gamma_{1} \neq \gamma_{2}$ and $\gamma_{1} \in \gamma_{2} G^{\prime}$. To see this, note that, for any $x \in N$, there is some $i \in\{1,2\}$, such that $\left\langle\gamma_{i} x\right\rangle$ has nontrivial projection to $G^{\prime}$ (with respect to the unique direct-product decomposition $\left.N=G^{\prime} \times\left(G^{\prime}\right)^{\perp}\right)$. Since $\left|G^{\prime}\right|$ is prime, this implies that the projection is all of $G^{\prime}$, so Lemma 3.6 tells us that $\left\langle\gamma_{i} x\right\rangle$ contains $G^{\prime}$. This establishes $\alpha_{2}^{1}$, which is equivalent to $\alpha_{2}^{2}$ (see remark 2.7(1)). This completes the proof of the claim.

Assume, for the moment, that $r=3$. Then, since $r \geq|\bar{a}| / 2$ and $|\bar{a}| \geq 6$, we must have $|\bar{a}|=6$. Here are two hamiltonian cycles in $\operatorname{Cay}(\bar{G} ; \bar{a}, \bar{b})$ that cover $S^{ \pm 1}$ :

$$
\left(b^{-1}, a^{-2}, b^{-4}, a^{-2}, b^{-1}, a^{3}, b^{2}, a, b^{-2}\right)
$$

and

$$
\left(b^{-1}, a^{-2}, b^{-1}, a, b^{-1}, a^{-1}, b^{-2}, a^{-1}, b^{-1}, a^{2}, b^{2}, a, b^{-2}\right)
$$

(see Figure 1). By using Lemma 3.3, we see that their voltages are

$$
b^{-1} a^{-2} b^{-4} a^{-2} b^{-1} a^{3} b^{2} a b^{-2}=b^{-6}[a, b]^{-(-10)}=b^{-6}[a, b]
$$

and

$$
b^{-1} a^{-2} b^{-1} a b^{-1} a^{-1} b^{-2} a^{-1} b^{-1} a^{2} b^{2} a b^{-2}=b^{-6}[a, b]^{-(-8)}=b^{-6}[a, b]^{2},
$$

respectively. So we may let $\gamma_{1}=b^{-6}[a, b]$ and $\gamma_{2}=b^{-6}[a, b]^{2}$.

We may now assume $r \geq 6$ (since $r$ is divisible by 3 ). Let

$$
I= \begin{cases}\{0,1\} & \text { if } r \neq|\bar{a}|, \\ \{1,2\} & \text { if } r=|\bar{a}| .\end{cases}
$$



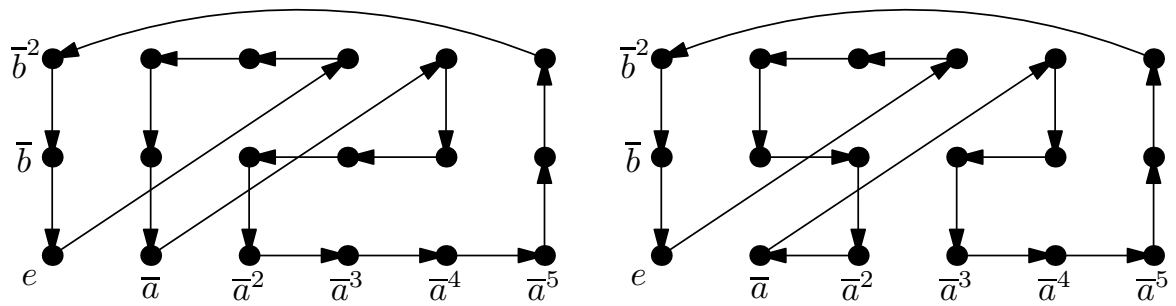

Figure 1: Two hamiltonian cycles in $\operatorname{Cay}(\bar{G} ;\{\bar{a}, \bar{b}\})$ when $m=r=3$.

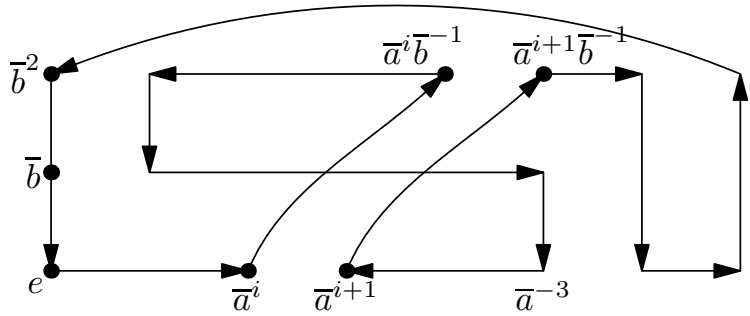

Figure 2: A hamiltonian cycle in Cay $(\bar{G} ;\{\bar{a}, \bar{b}\})$ when $m=3$ and $r \geq 6$.

Then, for $i \in I$, we have $0 \leq i \leq|\bar{a}|-4$, and $4 \leq r-i \leq|\bar{a}|-1$, so the walk $C_{i}=\left(a^{i}, b^{-1}, a^{-(|\bar{a}|-r+i-1)}, b^{-1}, a^{|\bar{a}|-4}, b^{-1}, a^{-(|\bar{a}|-i-4)}, b^{-1}, a^{r-i-3}, b^{-2}, a, b^{2}, a, b^{-2}\right)$ is as pictured in Figure 2. It is a hamiltonian cycle in $\operatorname{Cay}(\bar{G} ; \bar{a}, \bar{b})$ that covers $S^{ \pm 1}$. Furthermore, since

$$
a^{i} b^{-1} a^{-i}=b^{-1}\left(b a^{i} b^{-1} a^{-i}\right)=b^{-1}\left[b^{-1}, a^{-i}\right]=b^{-1}[b, a]^{i}=b^{-1}[a, b]^{-i},
$$

its voltage is of the form $h_{2}[a, b]^{-2 i}$, where $h_{2}$ is independent of $i$. Thus, we may let

$$
\left\{\gamma_{1}, \gamma_{2}\right\}=\left\{h_{2}[a, b]^{-2 i} \mid i \in I\right\} .
$$

Case 2. Assume $m \neq 3$. (Cf. [9, Case 4.3].) Since $m$ and $|\bar{a}|$ both have at least one odd prime divisor, we must have $m \geq 5$ and $|\bar{a}| \geq 5$. Let

$X=\left\{\begin{array}{cl}\left(b^{-(m-2)}, a, b^{m-3}, a^{|\bar{a}|-3}, b^{-1},\left(a^{-(|\bar{a}|-4)}, b^{-1}, a^{|\bar{a}|-4}, b^{-1}\right)^{(m-3) / 2}\right) & \text { if }|\bar{a}| \text { is odd, } \\ \left(b^{-1},\left(b^{-(m-3)}, a, b^{m-3}, a\right)^{(|\bar{a}| / 2)-1}, b^{-(m-2)}\right) & \text { if }|\bar{a}| \text { is even. }\end{array}\right.$

For each $i$ with $1 \leq i \leq\lfloor(|\bar{a}|-1) / 2\rfloor$, we have $1 \leq i \leq \min (r-1,|\bar{a}|-3)$ (since $r \geq|\bar{a}| / 2$ and $|\bar{a}| \geq 5$ ), so we may let

$$
C_{i}=\left(a^{i}, b^{-1}, a^{-(|\bar{a}|+i-r-1)}, X, a^{-(|\bar{a}|-i-2)}, b^{-1}, a^{r-i-1}, b^{-(m-1)}\right)
$$

(see Figures 3 and 4 ). Then $C_{i}$ is a hamiltonian cycle in $\operatorname{Cay}(\bar{G} ; \bar{a}, \bar{b})$. 


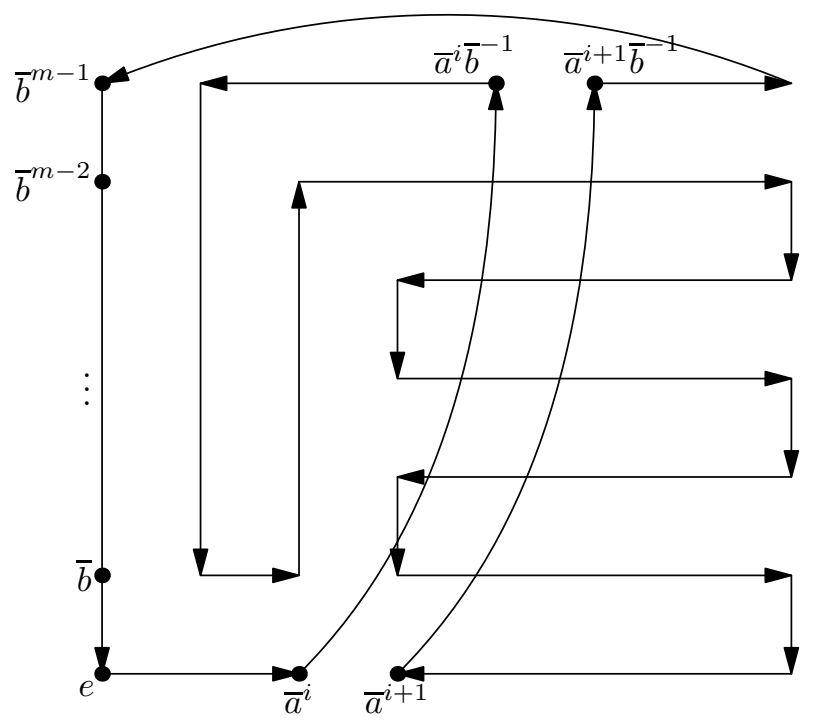

Figure 3: A hamiltonian cycle $C_{i}$ in $\operatorname{Cay}(\bar{G} ;\{\bar{a}, \bar{b}\})$ when $m=|\bar{G} /\langle\bar{a}\rangle|$ is odd.

Note that both possibilities for $X$ contain oriented edges labelled $a, b$, and $b^{-1}$. Furthermore, since $|\bar{a}|-i-2 \geq 1$, we see that $C_{i}$ also contains at least one oriented edge labelled $a^{-1}$. Therefore $C_{i}$ covers $\left\{a, b, a^{-1}, b^{-1}\right\}=S^{ \pm 1}$.

As in Case 1, the voltage $\Pi C_{i}$ of $C_{i}$ is of the form $h_{2}[a, b]^{-2 i}$, where $h_{2}$ is independent of $i$. Since $|\bar{a}| \geq\left|G^{\prime}\right|$ (see Lemma 3.4) and $\langle[a, b]\rangle=G^{\prime}$ (see Lemma 3.5), Lemma 3.8 (combined with Lemma 3.6) tells us that for any $x \in N$, we may choose $i$ so that $\left\langle\left(\Pi C_{i}\right) x\right\rangle$ contains $\left(G^{\prime}\right)^{2}$.

\section{The main induction step}

The induction step of our proof uses the following well-known gluing technique that is illustrated in Figure 5.

Definition 5.1. Let

- $C_{1}$ and $C_{2}$ be two vertex-disjoint oriented cycles in $\operatorname{Cay}(\bar{G} ; \bar{S})$,

- $g \in G$, and

- $a, s \in S$.

If

- $C_{1}$ contains the oriented edge $\bar{g}(s)$, and

- $C_{2}$ contains the oriented edge $\overline{g s a}\left(s^{-1}\right)$,

then we use $C_{1} \#_{s}^{a} C_{2}$ to denote the oriented cycle obtained from $C_{1} \cup C_{2}$ as in Figure 5, by

- removing the oriented edges $\bar{g}(s)$ and $\overline{g s a}\left(s^{-1}\right)$, and 


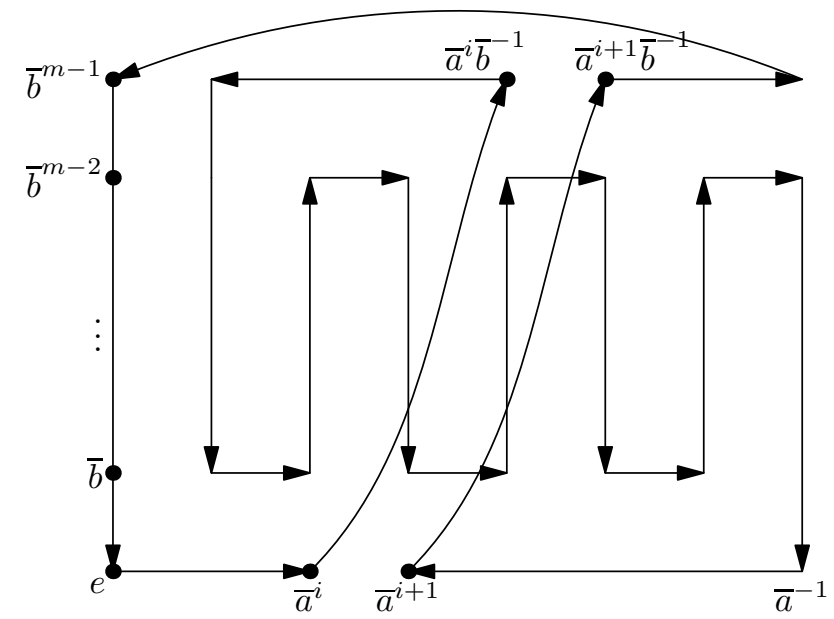

Figure 4: A hamiltonian cycle $C_{i}$ in $\operatorname{Cay}(\bar{G} ;\{\bar{a}, \bar{b}\})$ when $|\bar{a}|$ is even.

- inserting the oriented edges $\bar{g}(a)$ and $\overline{g s a}\left(a^{-1}\right)$.

This is called the connected sum of $C_{1}$ and $C_{2}$.

Lemma 5.2. If $C_{1}, C_{2}, g, s$, and $a$ are as in definition 5.1, and $N \subset Z(G)$, then

$$
\Pi\left(C_{1} \#_{s}^{a} C_{2}\right)=\left(\Pi C_{1}\right)\left(\Pi C_{2}\right)[a, s] .
$$

Proof. Write $C_{1}=\overline{g s}\left(s_{i}\right)_{i=1}^{m}$ and $C_{2}=\overline{g a}\left(t_{j}\right)_{j=1}^{n}$. Then

$$
C_{1} \#_{s}^{a} C_{2}=\overline{g s a}\left(a^{-1},\left(s_{i}\right)_{i=1}^{m-1}, a,\left(t_{j}\right)_{j=1}^{n-1}\right),
$$

so

$$
\begin{array}{rlr}
\Pi\left(C_{1} \#_{s}^{a} C_{2}\right) & =a^{-1}\left(\prod_{i=1}^{m-1} s_{i}\right) a\left(\prod_{j=1}^{n-1} t_{j}\right) \\
& =a^{-1}\left(\prod_{i=1}^{m} s_{i}\right) s_{m}^{-1} a\left(\prod_{j=1}^{n} t_{j}\right) t_{n}^{-1} & \\
& =a^{-1}\left(\Pi C_{1}\right) s^{-1} a\left(\Pi C_{2}\right) s & \\
& =\left(\Pi C_{1}\right)\left(\Pi C_{2}\right) a^{-1} s^{-1} a s & \left(\Pi C_{i} \in N \subset Z(G)\right) \\
& =\left(\Pi C_{1}\right)\left(\Pi C_{2}\right)[a, s] .
\end{array}
$$

Corollary 5.3. Assume

- $2 \leq k<\ell$, and (to eliminate some subscripts) $m=m_{k+1}$ and $a=\sigma_{k+1}$,

- $\pi_{1}, \pi_{2}, \ldots, \pi_{m}$ are elements of $\mathcal{V}_{k}$, 


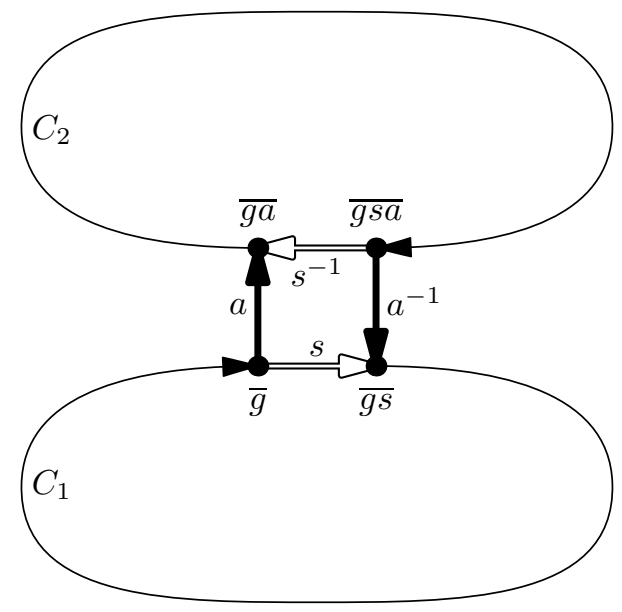

Figure 5: $C_{1}$ and $C_{2}$ are merged into a single cycle by replacing the two white edges labelled $s$ and $s^{-1}$ with the two black edges labelled $a$ and $a^{-1}$.

- $s_{1}, s_{2}, \ldots, s_{m-1}$ are elements of $S_{k}$, and, for each $i$, a choice $s_{i}^{*} \in\left\{s_{i}^{ \pm 1}\right\}$ has been made in such a way that if $s_{i+1}=s_{i}$, then $s_{i+1}^{*}=s_{i}^{*}$, and

- $N \subset Z(G)$.

Then there is a hamiltonian cycle in Cay $\left(\overline{G_{k+1}} ; \overline{S_{k+1}}\right)$ that covers $S_{k+1}^{ \pm 1}$, and whose voltage is

$$
\left(\prod_{i=1}^{m} \pi_{i}\right)\left(\prod_{i=1}^{m-1}\left[a, s_{i}^{*}\right]\right) .
$$

Proof. For each $i$, let $C_{i}$ be an oriented hamiltonian cycle in Cay $\left(\overline{G_{k}} ; \overline{S_{k}}\right)$ that covers $S_{k}^{ \pm 1}$, and has voltage $\pi_{i}$. We inductively construct sequences $\left(g_{i}\right)_{i=1}^{m}$ and $\left(x_{i}\right)_{i=1}^{m}$ of elements of $G_{k}$, as follows.

Let $g_{1}=e$. Since $C_{1}$ covers $S_{k}^{ \pm 1}$, we know there is some $x_{1} \in G_{k}$, such that $a g_{1} C_{1}$ contains the oriented edge $\overline{a x_{1}}\left(s_{1}^{*}\right)$.

Now, suppose $g_{1}, x_{1}, g_{2}, x_{2}, \ldots, g_{i}, x_{i} \in G_{k}$ are given, such that the connected sum

$$
a g_{1} C_{1} \#_{s_{1}^{*}}^{a} a^{2} g_{2} C_{2} \#_{s_{2}^{*}}^{a} \cdots \#_{s_{i-1}^{*}}^{a} a^{i} g_{i} C_{i}
$$

exists, and contains the oriented edge $\overline{a^{i} x_{i}}\left(s_{i}^{*}\right)$. Since $C_{i+1}$ covers $S_{k}^{ \pm 1}$, we know that $C_{i+1}$ contains an oriented edge labelled $\left(s_{i}^{*}\right)^{-1}$, and a different oriented edge that is labelled $s_{i+1}^{*}$. Therefore, there exist $g_{i+1}, x_{i+1} \in G_{k}$, such that

$$
a^{i+1} g_{i+1} C_{i+1} \text { contains the oriented edges } \overline{a^{i+1} x_{i} s_{i}^{*}}\left(\left(s_{i}^{*}\right)^{-1}\right) \text { and } \overline{a^{i+1} x_{i+1}}\left(s_{i+1}^{*}\right) .
$$

The first of these edges is removed when we form the connected sum

$$
\left(a g_{1} C_{1} \#_{s_{1}^{*}}^{a} a^{2} g_{2} C_{2} \#_{s_{2}^{*}}^{a} \cdots \#_{s_{i-1}^{*}}^{a} a^{i} g_{i} C_{i}\right) \#_{s_{i}^{*}}^{a} a^{i+1} g_{i+1} C_{i+1},
$$


but the second edge remains, and will be used to form the next connected sum (unless $i+1=m)$.

Since each $C_{i}$ is a hamiltonian cycle in $\operatorname{Cay}\left(\overline{G_{k}} ; \overline{S_{k}}\right)$, the resulting connected sum

$$
a g_{1} C_{1} \#_{s_{1}^{*}}^{a} a^{2} g_{2} C_{2} \#_{s_{2}^{*}}^{a} \cdots \#_{s_{m-1}^{*}}^{a} a^{m} g_{m} C_{m}
$$

passes through all of the vertices in $\overline{a G_{k}} \cup \overline{a^{2} G_{k}} \cup \cdots \cup \overline{a^{m} G_{k}}$. That is, it passes through every element of $\overline{G_{k+1}}$, so it is a hamiltonian cycle in Cay $\left(\overline{G_{k+1}} ; \overline{S_{k+1}}\right)$. Its voltage is calculated by repeated application of Lemma 5.2.

To complete the proof, we verify that the hamiltonian cycle covers $S_{k+1}^{ \pm 1}$. Since each $C_{i}$ covers $S_{k}^{ \pm 1}$, the disjoint union

$$
a g_{1} C_{1} \cup a^{2} g_{2} C_{2} \cup \cdots \cup a^{m} g_{m} C_{m}
$$

contains (at least) $m$ disjoint pairs of edges labelled $s$ and $s^{-1}$, for each $s \in S_{k}$. Each invocation of the connected sum removes only one such pair, and the operation is performed only $m-1$ times, so at least one of the $m$ pairs must remain, for each $s \in S_{k}$. Therefore, the hamiltonian cycle covers $S_{k}^{ \pm 1}$. Also, the cycle certainly covers $a^{ \pm 1}$, since each invocation of the connected sum inserts a pair of edges labelled $a$ and $a^{-1}$. Hence, the hamiltonian cycle covers $S_{k}^{ \pm 1} \cup\left\{a^{ \pm 1}\right\}=S_{k+1}^{ \pm 1}$.

We can now prove the main result of this section. (Recall that the condition $\alpha_{k}^{\epsilon}$ is defined in Section 2.)

Proposition 5.4. Assume $|N|$ is square-free and $\left|G_{k+1}^{\prime} / G_{k}^{\prime}\right|$ is odd. Then

1. $\alpha_{k}^{1} \Rightarrow \alpha_{k+1}^{1}$, and

2. $\alpha_{k}^{2+} \Rightarrow \alpha_{k+1}^{2+}$ if $|[s, t]|$ is even for all $s, t \in S_{k+1}$ with $s \neq t$.

Proof. For convenience, let $m=m_{k+1}$ and $a=\sigma_{k+1}$. Let $h_{k} \in N$ be as in $\left(\alpha_{k}^{\epsilon}\right)$, and choose an oriented hamiltonian cycle $C$ in $\operatorname{Cay}\left(\overline{G_{k}} ; \overline{S_{k}}\right)$ that covers $S_{k}^{ \pm 1}$, and has its voltage in $h_{k}\left(G_{k}^{\prime}\right)^{\epsilon}$. There is no harm in assuming that the voltage is precisely $h_{k}$. Let

$$
h_{k+1}=\left(h_{k}\right)^{m}\left[a, \sigma_{1}\right]^{m-1} .
$$

Given any $x \in N$, corollary 3.11 provides a sequence $\left(s_{i}\right)_{i=1}^{m-1}$ of elements of $S_{k}$, and $s_{i}^{*} \in\left\{s_{i}^{ \pm 1}\right\}$ for each $i$, such that $s_{i+1}^{*}=s_{i}^{*}$ whenever $s_{i+1}=s_{i}$, and

$$
\left\langle x\left(h_{k}\right)^{m} \prod_{i=1}^{m-1}\left[a, s_{i}^{*}\right],\left(G_{k}^{\prime}\right)^{\epsilon}\right\rangle \text { contains }\left(G_{k+1}^{\prime}\right)^{\epsilon} .
$$

From $\left(\alpha_{k}^{\epsilon}\right)$ we know there exists $\pi \in \mathcal{V}_{k} \cap h_{k}\left(G_{k}^{\prime}\right)^{\epsilon}$, such that, if we let

$$
\gamma=\pi\left(h_{k}\right)^{m-1} \prod_{i=1}^{m-1}\left[a, s_{i}^{*}\right]
$$

then $\langle x \gamma\rangle$ contains $\left(G_{k}^{\prime}\right)^{\epsilon}$. Since $\pi \equiv h_{k}\left(\bmod \left(G_{k}^{\prime}\right)^{\epsilon}\right)$, combining this with (5.5) shows that $\langle x \gamma\rangle$ contains $\left(G_{k+1}^{\prime}\right)^{\epsilon}$. Also, since we are assuming $\left|\left[a, s_{i}^{*}\right]\right|$ is even if $\epsilon=2$, we have $\left[a, s_{i}^{*}\right] \equiv\left[a, \sigma_{1}\right]\left(\bmod \left(G_{k+1}^{\prime}\right)^{\epsilon}\right)$ for all $i$, so

$$
\gamma \in\left(h_{k}\right)^{m}\left[a, \sigma_{1}\right]^{m-1}\left(G_{k+1}^{\prime}\right)^{\epsilon}=h_{k+1}\left(G_{k+1}^{\prime}\right)^{\epsilon} .
$$


Furthermore, corollary 5.3 tells us that there is a hamiltonian cycle in Cay $(\bar{G} ; \bar{S})$ whose voltage is $\gamma$, and this hamiltonian cycle covers $S^{ \pm 1}$. This establishes $\alpha_{k+1}^{\epsilon}$.

Now, if $\epsilon=2$, then our assumptions imply that $\left|h_{k}\right|$ and $\left|\left[a, \sigma_{1}\right]\right|$ are both even. Since $m$ and $m-1$ are of opposite parity, this implies that $\left|h_{k+1}\right|$ is even, so $\left\langle h_{k},\left(G_{k+1}^{\prime}\right)^{2}\right\rangle$ contains $G_{k+1}^{\prime}$. This establishes $\alpha_{k+1}^{2+}$.

\section{Combining the base case with the induction step}

Recall that the condition $\alpha_{k}^{\epsilon}$ is defined in Section 2.

Corollary 6.1. Assume $|N|$ is square-free and $\ell \geq 2$. If $\left|G^{\prime}\right|$ is odd, then $\alpha_{\ell}^{1}$ is true unless $\left|G^{\prime}\right|=|\bar{s}|=3$ for all $s \in S$.

Proof. Assume it is not the case that $\left|G^{\prime}\right|=|\bar{s}|=3$ for all $s \in S$. Then we may assume (by permuting the elements of $S$ ) that either $\left|G_{2}^{\prime}\right| \neq 3$ or $\left|\sigma_{1}\right| \neq 3$. Therefore proposition 4.1 tells us that $\alpha_{2}^{2}$ is true. Also, since $\left|G^{\prime}\right|$ is odd, we have $\alpha_{2}^{2} \Leftrightarrow \alpha_{2}^{1}$ (see remark 2.7(1)), so $\alpha_{2}^{1}$ is true. Then repeated application of proposition 5.4(1) establishes $\alpha_{\ell}^{1}$.

Proposition 6.2. Assume $|N|$ is square-free and $\ell \geq 3$. If $\left|G^{\prime}\right|$ is even, then:

1. $\alpha_{\ell}^{1}$ is true if there exist $s, t \in S$, such that $|[s, t]|$ is odd and $s \neq t$.

2. $\alpha_{\ell}^{2+}$ is true if $|[s, t]|$ is even for all $s, t \in S$ with $s \neq t$.

Proof. Since $\left|G^{\prime}\right|$ is even, we may assume (by permuting the elements of $S$ ) that $\left|\left[\sigma_{3}, \sigma_{1}\right]\right|$ is even. It suffices to prove $\alpha_{3}^{1}$ or $\alpha_{3}^{2+}$ (as appropriate), for then repeated application of proposition 5.4 establishes the desired conclusion. Thus, we may assume $\ell=3$, so $G_{3}=$ $G$. Let $m=m_{3}$ and $a=\sigma_{3}=\sigma_{\ell}$.

By permuting the elements of $S$, we may assume that either:

odd case: $\left|\left[\sigma_{3}, \sigma_{2}\right]\right|$ is odd, or

even case: $|[s, t]|$ is even for all $s, t \in S$ with $s \neq t$.

Furthermore, in the even case, we may assume that either:

even subcase: $\left\langle\overline{\sigma_{1}}, \overline{\sigma_{2}}\right\rangle$ has even index in $\bar{G}$, or

odd subcase: $\langle\bar{s}, \bar{t}\rangle$ has odd index in $\bar{G}$, for all $s, t \in S$, such that $s \neq t$.

Since $\left|\left[\sigma_{3}, \sigma_{1}\right]\right|$ is even, we know $\left|\overline{\sigma_{1}}\right|$ is even (see Lemma 3.4). In particular, we have $\left|\overline{\sigma_{1}}\right| \neq 3$, so proposition 4.1 tells us that $\alpha_{2}^{2}$ is true.

We now use a slight modification of the proof of proposition 5.4. Let $h_{2} \in N$ be as in $\left(\alpha_{k}^{\epsilon}\right)$ (with $k=\epsilon=2$ ), and choose an oriented hamiltonian cycle $C$ in $\operatorname{Cay}\left(\overline{G_{2}} ; \overline{S_{2}}\right)$ that covers $S_{2}^{ \pm 1}$, and has its voltage in $h_{2}\left(G_{2}^{\prime}\right)^{2}$. There is no harm in assuming that the voltage is precisely $h_{2}$.

Since $\left|\overline{\sigma_{1}}\right|$ is even, we know $\left|\overline{G_{2}}\right|$ is even. Therefore, Lemma 3.15 provides a hamiltonian cycle $C^{\prime}$ in $\operatorname{Cay}\left(\overline{G_{2}} ; \overline{S_{2}}\right)$, such that $\Pi C^{\prime}$ is a generator of $G_{2}^{\prime}$. Let

$$
h^{\prime}=\left\{\begin{aligned}
\Pi C^{\prime} & \text { in the odd subcase of the even case } \\
h_{2} & \text { in all other cases. }
\end{aligned}\right.
$$

Let $h_{3}=\left(h_{2}\right)^{m-1} h^{\prime}\left[a, \sigma_{1}\right]^{m-1}$. 
Given any $x \in N$, corollary 3.11 provides a sequence $\left(s_{i}\right)_{i=1}^{m-1}$ of elements of $S_{2}$, and $s_{i}^{*} \in\left\{s_{i}^{ \pm 1}\right\}$ for each $i$, such that $s_{i+1}^{*}=s_{i}^{*}$ whenever $s_{i+1}=s_{i}$, and

$$
\left\langle x\left(h_{2}\right)^{m-1} h^{\prime} \prod_{i=1}^{m-1}\left[a, s_{i}^{*}\right],\left(G_{2}^{\prime}\right)^{2}\right\rangle \text { contains }\left(G^{\prime}\right)^{2} .
$$

Furthermore, in the odd case, the choices can be made so that (6.3) holds with $G^{\prime}$ in the place of $\left(G^{\prime}\right)^{2}$.

From $\alpha_{2}^{2}$, we know there exists $\pi \in \mathcal{V}_{2} \cap h_{2}\left(G_{2}^{\prime}\right)^{2}$, such that, if we let

$$
\gamma=\pi\left(h_{2}\right)^{m-2} h^{\prime} \prod_{i=1}^{m-1}\left[a, s_{i}^{*}\right]
$$

then

$$
\langle x \gamma\rangle \text { contains }\left(G^{\prime}\right)^{2} \text {. }
$$

It is clear from the definitions that $\gamma \in h_{3} G_{3}^{\prime}$. Furthermore, we have $\gamma \in h_{3}\left(G_{3}^{\prime}\right)^{2}$ in the even case.

corollary 5.3 tells us that there is a hamiltonian cycle in Cay $(\bar{G} ; \bar{S})$ whose voltage is $\gamma$, and this hamiltonian cycle covers $S^{ \pm 1}$. We now consider various cases individually.

Case 1. The odd case. Recall that, in this case, (6.3) holds with $G^{\prime}$ in the place of $\left(G^{\prime}\right)^{2}$. Since $\pi \equiv h_{2}\left(\bmod \left(G_{2}^{\prime}\right)^{2}\right)$, combining this with (6.4) shows that $\langle x \gamma\rangle$ contains all of $G^{\prime}$. This establishes $\alpha_{3}^{1}$.

Case 2. The even subcase of the even case. In this subcase, we know $m$ is even, $h^{\prime}=h_{2}$, and $\left|\left[a, \sigma_{1}\right]\right|$ is even. Since $h_{3}=\left(h_{2}\right)^{m}\left[a, \sigma_{1}\right]^{m-1}$, we see that $\left|h_{3}\right|$ is even, so $\left\langle h_{3},\left(G_{3}^{\prime}\right)^{2}\right\rangle$ contains $G_{3}^{\prime}$. This establishes $\alpha_{3}^{2+}$.

Case 3. The odd subcase of the even case. In this subcase, we know $m-1$ is even, and $h^{\prime}=\Pi C^{\prime}$ has even order. Therefore $\left|h_{3}\right|$ is even, so $\left\langle h_{3},\left(G_{3}^{\prime}\right)^{2}\right\rangle$ contains $G_{3}^{\prime}$. This establishes $\alpha_{3}^{2+}$.

We can now establish our main theorem:

Proof of theorem 1.2. We may assume:

- $\ell \geq 3$, for otherwise proposition 3.16 applies.

- $|G|$ is not a power of 3 , for otherwise theorem 3.13 applies.

Let $S$ be a minimal generating set of $G$, and let $N=G^{\prime}$. Note that $\bar{S}$ is a minimal generating set of $\bar{G}$ (because $G^{\prime}$ is contained in the Frattini subgroup $\Phi(G)$ [8, Thm. 10.4.3]).

We claim there is a hamiltonian cycle in $\operatorname{Cay}(\bar{G} ; \bar{S})$ whose voltage generates $G^{\prime}$. While proving this, there is no harm in assuming that $\left|G^{\prime}\right|$ is square-free (see Lemma 3.14). Also note that, since $|G|$ is not a power of 3 , we cannot have $\left|G^{\prime}\right|=|\bar{s}|=3$ for all $s \in S$. Then, by applying either corollary 6.1 or proposition 6.2 (depending on the parity of $\left|G^{\prime}\right|$ ), we obtain either $\alpha_{\ell}^{1}$ or $\alpha_{\ell}^{2+}$. Each of these yields the desired hamiltonian cycle in Cay $(\bar{G} ; \bar{S})$ (see Lemma 2.6).

Now that the claim has been verified, the Factor Group Lemma (2.5) provides a hamiltonian cycle in $\operatorname{Cay}(G ; S)$. 


\section{References}

[1] B. Alspach, C. C. Chen and M. Dean, Hamilton paths in Cayley graphs on generalized dihedral groups, Ars Math. Contemp. 3 (2010), 29-47. MR 2592513

[2] B. Alspach and C. Q. Zhang, Hamilton cycles in cubic Cayley graphs on dihedral groups, Ars Combin. 28 (1989), 101-108. MR 1039136

[3] C. C. Chen and N. Quimpo, On strongly hamiltonian abelian group graphs, in: K. L. McAvaney, ed., Combinatorial Mathematics VIII (Proceedings, Geelong, Australia 1980), Springer-Verlag, Berlin, 1981, pp. 23-24. MR 0641233

[4] S. J. Curran and J. A. Gallian, Hamiltonian cycles and paths in Cayley graphs and digraphs-a survey, Discrete Math. 156 (1996) 1-18. MR 1405010

[5] E. Durnberger, Connected Cayley graphs of semidirect products of cyclic groups of prime order by abelian groups are Hamiltonian, Discrete Math. 46 (1983), 55-68. MR 0708162

[6] E. Durnberger, Every connected Cayley graph of a group with prime order commutator group has a Hamilton cycle, in: B. Alspach and C. Godsil, eds., Cycles in Graphs (Burnaby, B.C., 1982), North-Holland, Amsterdam, 1985, pp. 75-80. MR 0821506

[7] D. Gorenstein, Finite Groups, Chelsea, New York, 1980, MR 0569209

[8] M. Hall, Jr., Theory of Groups, Macmillan, New York, 1959. MR 0103215

[9] K. Keating and D. Witte, On Hamilton cycles in Cayley graphs with cyclic commutator subgroup, in: B. Alspach and C. Godsil, eds., Cycles in Graphs (Burnaby, B.C., 1982), NorthHolland, Amsterdam, 1985, pp. 89-102. MR 0821508

[10] K. Kutnar and D. Marušič, Recent trends and future directions in vertex-transitive graphs, Ars Math. Contemp. 1 (2008), 112-125. MR 2443319

[11] K. Kutnar, D. Marušič, D. W. Morris, J. Morris and P. Šparl, Hamiltonian cycles in Cayley graphs whose order has few prime factors, Ars Math. Contemp. 5 (2012), 27-71. MR 2853700

[12] D. Marušič, Hamiltonian circuits in Cayley graphs, Discrete Math. 46 (1983) 49-54. MR 0708161

[13] D. W. Morris, 2-generated Cayley digraphs on nilpotent groups have hamiltonian paths, Contrib. Discrete Math. 7 (2012), 41-47.

[14] I. Pak and R. Radoičić, Hamiltonian paths in Cayley graphs, Discrete Math. 309 (2009), 55015508. MR 2548568

[15] D. Witte, On Hamiltonian circuits in Cayley diagrams, Discrete Math. 38 (1982), 99-108. MR 0676525

[16] D. Witte, Cayley digraphs of prime-power order are Hamiltonian, J. Combin. Theory Ser. B 40 (1986), 107-112. MR 0830597

[17] D. Witte and J. A. Gallian, A survey: Hamiltonian cycles in Cayley graphs, Discrete Math. 51 (1984) 293-304. MR 0762322 